\title{
Corrigendum: Telomere protection by mammalian Pot1 requires interaction with Tpp1
}

Dirk Hockemeyer, Wilhelm Palm, Tobias Else, Jan-Peter Daniels, Kaori K Takai, Jeffrey Z-S Ye, Catherine E Keegan, Titia de Lange \& Gary D Hammer

Nat. Struct. Mol. Biol. 14, 754-761 (2007); published online 15 July 2007; corrected after print 24 November 2008

In the version of this article initially published, one of the US National Institutes of Health grant numbers was incorrect. The correct acknowledgment should be: "US National Institutes of Health to T.d.L. (GM49046 and AG016642)." The error has been corrected in the HTML and PDF versions of the article.

\section{Corrigendum: Integration of an electric-metal sensory experience in the Slo1 BK channel}

Frank T Horrigan \& Toshinori Hoshi

Nat. Struct. Mol. Biol. 15, 1130-1132 (2008); corrected after print 6 May 2009

In the version of this article initially published, the incorrect PDB code for the MthK open channel structure was provided in the legend to Figure 1. The correct PDB code for this structure is 1LNQ. The error has been corrected in the HTML and PDF versions of the article. 